Pacific Journal of Mathematic 


\title{
POLYNOMIAL RINGS OVER FINITE DIMENSIONAL RINGS
}

\author{
ROBERT C. SHOCK
}

\begin{abstract}
A ring is right finite dimensional if it contains no infinite direct sum of nonzero right ideals. We prove that polynomial over finite dimensional rings are finite dimensional rings. The (Goldie) dimension of a ring is unaffected by adjoining to it an arbitrary number of indeterminates. Several applications are given.
\end{abstract}

1. Introduction. In $1960 \mathrm{~A}$. W. Goldie gave necessary and sufficient conditions for a ring to have a classical ring of right quotients which is a semiprime Artinian ring [1]. Following Goldie, a ring is right finite dimensional if it does not contain an infinite direct sum of nonzero right ideals. The class of right finite dimensional rings properly contains the class of right Noetherian rings. The Hilbert Basis Theorem states that if $R$ is a right Noetherian ring then the polynomial ring in a finite number of indeterminates over $R$ is also a right Noetherian ring. We extend this theorem to finite dimensional rings. Furthermore, the requirement of a finite number of indeterminates is dropped. We prove that if $R$ is a right finite dimensional ring then the polynomial ring in an arbitrary number of indeterminates over $R$ is also a right finite dimensional ring. We cite one application. An order theorem of A. W. Goldie is generalized to polynomial rings. We also prove that if a ring $R$ has a classical ring of right quotients which is a semiprime Artinian ring, then so does every polynomial ring over $R$.

Throughout this paper let $R$ always denote an associate ring which need not have an identity. For a subring $K$ of $R$ let $K\left[x_{1}, x_{2}, \cdots\right]$ denote the ring obtained by adjoining an arbitrary number of indeterminates $x_{1}, x_{2}, \cdots$ which commute with all elements of $K$ and with each other. Let $Z(S)$ denote the right singular ideal of the ring $S[2]$.

For a right finite dimensional ring $R$ there exists an integer $n$ such that $R$ contains a direct sum of $n$ - summands and the number of summands of any other direct sum in $R$ is at most $n$. This unique integer $n$ is called the dimension of $R$ and we write $\operatorname{dim} R=n$. Theorem 2.6 states that $\operatorname{dim} R=n$ if and only if $\operatorname{dim}\left(R\left[x_{1}, x_{2}, \cdots\right]\right)=$ $n$. In $\S 3$ we prove that $R$ is a semiprime Goldie ring if and only if the same is true of $R\left[x_{1}, x_{2}, \cdots\right]$. We also generalize to polynomial rings an order theorem of L. Small: If a ring $R$ has a classical ring of right quotients which is Artinian then so does $R\left[x_{1}, x_{2}, \cdots\right]$ 
(Theorem 3.7).

2. The main theorems. In this section we prove our main results. Theorem 2.6 states that $\operatorname{dim} R=\operatorname{dim} R\left[x_{1}, x_{2}, \cdots\right]$ for any right finite dimensional ring $R$. Theorem 2.7 states that $Z\left(R\left[x_{1}\right.\right.$, $\left.\left.x_{2}, \cdots\right]\right)=Z(R)\left[x_{1}, x_{2}, \cdots\right]$ for any ring $R$.

We denote the right annihilator of a subset $S$ of $R$ by $r(S)$, that is, $r(S)=\{y \in R: s y=0$ for all $s \in S\}$.

Imbed a ring $R$ into a ring with an identity in the "standard way" (this follows Dorroh). We denote this ring by $R^{1}$. For $b \in R$ we equate $b R^{1}$ with the principal right ideal generated by the element $b$ in $R$.

Let $R=\{x, 0\}$ where $x+x=x^{2}=0$. In $R^{1}$ the $\operatorname{sum}(x, 0) R^{1}+$ $(0,2) R^{1}$ is direct. Hence $\operatorname{dim} R=1 \neq \operatorname{dim} R^{1}=2$. We proceed under the assumption that a ring $R$ need not have an identity.

Lemma 2.1. Let $a_{1}, \cdots, a_{n}$ be in $R$. Then either $r\left(a_{n}\right)=r\left(a_{j}\right)$ for all $j$ where $1 \leqq j \leqq n$ or there exists $b \in R$ and an index $j$ where $1 \leqq j \leqq n$ such that $a_{j} b \neq 0$ and $r\left(a_{j} b\right)=r\left(a_{k} b\right)$ whenever $a_{k} b \neq 0$ where $1 \leqq k \leqq n$.

Proof. Assume all the right annihilators $r\left(a_{k}\right)$ are not the same. Consider the set of right annihilators $r\left(a_{i}\right)$ subject to $a_{j} \neq 0$ where $1 \leqq i \leqq n$. If two of these annihilators are different say $r\left(a_{k_{1}}\right)$ and $r\left(a_{k_{2}}\right)$ then choose $x \in r\left(a_{k_{1}}\right)$ and $x \notin r\left(a_{k_{2}}\right)$. Hence $a_{k_{2}} x \neq 0$ and $a_{k_{1}}=$ 0 . The new set of annihilators $r\left(a_{k} x\right)$ subject to $a_{k} x \neq 0$ has fewer members than the original set $r\left(a_{i}\right)$ subject to $a_{i} \neq 0$ where $1 \leqq i \leqq n$. We repeat this process until we obtain a multiplier $b$ in $R$ and an index $j$ such that Lemma 2.1 is satisfied.

Let $0 \neq g(x)=a_{0}+\cdots+a_{n} x^{n}$ be in $R[x]$. It is understood that $a_{n} \neq 0$. If $r\left(a_{n}\right)=r\left(a_{j}\right)$ in $R$ whenever $a_{j} \neq 0$ where $1 \leqq j \leqq n$ then we say that the right annililators of the coefficients of $g(x)$ are equal.

Proposition 2.2. For a nonzero polynomial $p(x)$ in $R[x]$ there exists $b \in R^{1}$ such that the right annihilators of the coefficients of the nonzero polynomial $p(x) b$ are equal.

Proof. Let $p(x)=a_{0}+\cdots+a_{n} x^{n}$. If $r\left(a_{n}\right)=r\left(a_{k}\right)$ where $a_{k} \neq 0$ and $1 \leqq k \leqq n$ then let $b=(0,1)$ in $R^{1}$ and the proposition follows. Suppose $r\left(a_{n}\right) \neq r\left(a_{k}\right)$ for some $a_{k} \neq 0$ and $1 \leqq k \leqq n$. By the previous lemma there is an element $d \in R$ and an index $j$ such that $a_{j} d \neq 0$ and $r\left(a_{j} d\right)=r\left(a_{h} d\right)$ whenever $a_{h} d \neq 0$ and $1 \leqq h \leqq n$. Let $b=(d, 0) \in$ $R^{1}$. Then $p(x) b$ satisfies the proposition. 
LEMMA 2.3. Let $p(x)=a_{k} x^{k}+\cdots+a_{k+n} x^{k+n}$ be a polynomial in $R[x]$ where $k \geqq 0$. Assume that $a_{k} \neq 0$ and $r\left(a_{k}\right) \subseteq r\left(a_{k+i}\right)$ for $1 \leqq$ $i \leqq n$. Let $q(x)=b_{0}+b_{1} x+\cdots+b_{m} x^{m}$ be in $R[x]$. Then $p(x) q(x)=$ 0 if and only if the coefficients $b_{0}, b_{1}, \cdots, b_{n}$ are in $r\left(a_{k+i}\right)$ for $1 \leqq$ $i \leqq n$.

Proof. Let $h(x)=p(x) q(x)=c_{j} x^{k}+\cdots+c_{k+n+m} x^{k+n+m}$ where $c_{k}=$ $a_{k} b_{0}, \cdots, c_{k+n+m}=a_{k+n} b_{m}$. Clearly $c_{k}=0$ implies $b_{0} \in r\left(a_{j}\right)$ and hence $b_{0} \in r\left(a_{k+1}\right)$ for $1 \leqq i \leqq n$. Now $0=c_{1}=a_{k+1} b_{0}+a_{k} b_{1}$ forces $b_{1} \in r\left(a_{k}\right)$ since $b_{0} \in r\left(a_{k+1}\right)$. Hence $b_{1} \in r\left(a_{k+1}\right)$ for $1 \leqq i \leqq n$. It follows that $0=c_{2}=a_{k+2} b_{0}+a_{k+1} b_{1}+a_{k} b_{2}$ implies $b_{2} \in r\left(a_{k}\right)$. Continuing in this fashion each $b_{j} \in r\left(a_{k}\right)$ for $1 \leqq j \leqq m$. Since $r\left(a_{k}\right) \leqq r\left(a_{k+i}\right)$ for $1 \leqq i \leqq n$ the implication follows. The reverse implication is clear.

A right ideal $K$ is said to be uniform in $R$ if $x$ and $y$ are in $K-(0)$ imply $x R^{1} \cap y R^{1} \neq(0)$.

Proposition 2.4. A right ideal $K$ is uniform in $R$ if and only if $K[x]$ is uniform in $R[x]$.

Proof. Assume that $K[x]$ is not a uniform right ideal in $R[x]$. Suppose that the sum $p(x) R[x]^{1}+q(x) R[x]^{1}$ is direct, where $p(x)$ and $q(x)$ belong to $K[x]-(0)$. By Proposition 2.2, we can choose $p(x)$ [and $q(x)$ ] such that the right annihilators of the coefficients of $p(x)$ [and $q(x)]$ are equal. Let $p(x)=a_{0}+a_{1} x+\cdots+a_{k} x^{k}$ and $q(x)=$ $b_{0}+b_{1} x+\cdots+b_{n} x^{n}$, where $a_{i} \neq 0$ and $b_{j} \neq 0$ imply $r\left(a_{k}\right)=r\left(a_{i}\right)$ and $r\left(b_{n}\right)=r\left(b_{j}\right)$ for $1 \leqq i \leqq k$ and $1 \leqq j \leqq n$. Furthermore, we may assume $n \geqq k$. Since the nonnegative integers are well ordered, we may assume that for any other direct sum of the form $p(x) R[x]^{1}+$ $t(x) R[x]^{1}$ we have degree $t(x) \geqq$ degree $q(x)$, where $t(x) \in K[x]-(0)$ and the right annihilators of the coefficients of $t(x)$ are equal, Recall next that $a_{k}$ and $b_{n}$ are in a uniform right ideal and there exist $y$ and $z$ in $R^{1}$ such that $0 \neq a_{k} y=b_{n} z$. Let $h(x)=p(x) y x^{n-k}+q(x)(-z)$. Clearly degree of $h(x)<$ degree of $q(x)$. Also $h(x) \neq 0$; otherwise the sum $p(x) R[x]^{1}+q(x) R[x]^{1}$ would not be direct. We claim the sum $h(x) R[x]^{1}+p(x) R[x]^{1}$ is not direct. The right annihilators of the coefficients of $h(x) b \neq 0$ are equal, where $b$ is an appropriate element in $R^{1}$; such a $b$ exists by Proposition 2.2. Clearly degree of $h(x) b<$ degree of $q(x)$. If $h(x) R[x]^{1}+p(x) R[x]^{1}$ were direct, then $[h(x) b] R[x]^{1}+$ $p(x) R[x]^{1}$ would be direct, a contradiction of the choosing of the degree of $q(x)$. Hence $0 \neq h(x) m(x)=p(x) g(x)$ for some $m(x)$ and $g(x)$ in $R[x]^{1}$. If $q(x)(-z) m(x)=0$, then the coefficients of $m(x)$ are in $r\left(b_{n} z\right)$ by Lemma 2.3. But $r\left(b_{n} z\right)=r\left(a_{k} y\right)$ implies that $p(x) y x^{n-k} m(x)=0$, again by Lemma 2.3. Thus $0 \neq h(x) m(x)=\left[p(x) y x^{n-k}+q(x)(-z)\right] m(x)=$ 
0 , a contradiction. We conclude that $q(x)(-z) m(x) \neq 0$. This forces $q(x)(-z) m(x)=p^{\prime}(x) g(x)-p(x) y x^{k-k} m(x)$, which contradicts our assumption that the sum $q(x) R[x]^{1}+p(x) R[x]^{1}$ was direct. We conclude that $K[x]$ is a uniform right ideal. The reverse implication is clear.

A right ideal is said to be essential in $R$ if it has nonzero intersection with each nonzero right ideal of $R$. Assume $K$ is an essential right ideal. For nonzero elements $a_{1}, a_{2}, \cdots, a_{n}$ in $R$ there does exist $b \in R^{1}$ such that $a_{i} b \in K-(0)$ for some $i$ where $1 \leqq i \leqq n$ and $a_{j} b \in$ $K$ for $1 \leqq j \leqq n$. This follows from the definition.

Proposition 2.5. A ring $R$ is right finite dimensional if and only if the polynomial ring $R[x]$ is. Furthermore, $\operatorname{dim} R=\operatorname{dim} R[x]$.

Proof. Assume $R$ is right finite dimensional. There exists a finite set of nonzero uniform right ideals $\left\{L_{1}, L_{2}, \cdots, L_{k}\right\}$ such that the sum $L^{\prime}=L_{1}+\cdots+L_{k}$ is direct and $L^{\prime}$ is essential in $R$. Clearly, $L^{\prime}[x]=L_{1}[x]+\cdots+L_{k}[x]$ and this sum is direct. For $1 \leqq i \leqq k$ each $L_{i}[x]$ is uniform in $R[x]$ by Proposition 2.4. We claim that $L^{\prime}$ is essential in $R$ implies that $L^{\prime}[x]$ is essential in $R[x]$. Let $p(x)$ be a nonzero polynomial in $R[x]$. Then there is some $b \in R^{1}$ such that $p^{\prime}(x) b \neq 0$ and each coefficient of $p(x) b$ belongs to $L^{\prime}$. Hence $p(x) b \in$ $L^{\prime}[x]$. Also note that $\operatorname{dim} R=\operatorname{dim} R[x]$. The reverse implication is clear.

THEOREM 2.6. A ring $R$ is right finite dimensional if and only if $R\left[x_{1}, x_{2}, \cdots\right]$ is. Furthermore, $\operatorname{dim} R=\operatorname{dim} R\left[x_{1}, x_{2}, \cdots\right]$.

Proof. Assume that $\operatorname{dim} R=n$. First, $\operatorname{dim} R=\operatorname{dim} R\left(\left[x_{1}, \cdots, x_{k}\right]\right)$ for any nonnegative integer $k$. This follows by finite induction on the number of indeterminates and by Proposition 2.5. Let $S=$ $R\left[x_{1}, x_{2}, \cdots\right]$. Suppose that the sum $p_{1} S^{1}+\cdots+p_{n} S^{1}+p_{n \div 1} S^{1}$ is direct where $p_{i} \in S$ for $1 \leqq i \leqq n+1$. Each term of each polynomial $p_{i}$ for $1 \leqq i \leqq n+1$ involves only a finite number of indeterminates and for $1 \leqq i \leqq n+1$ each $p_{i} \in R\left[x_{1}^{\prime}, \cdots, x_{k}^{\prime}\right]$ where $x_{1}^{\prime}, x_{2}^{\prime}, \cdots, x_{k}^{\prime}$ is an appropriate finite subset of the indeterminates $x_{1}, x_{2}, \cdots$. Let $T=R\left[x_{1}^{\prime}, \cdots, x_{k}^{\prime}\right]$ and the sum $p_{1} T^{1}+\cdots+p_{n+1} T^{1}$ is direct. This forces $\operatorname{dim} T>n=\operatorname{dim} R$, a contradiction. Clearly $\operatorname{dim} S \geqq \operatorname{dim} R$ and hence, $\operatorname{dim} R=\operatorname{dim} S$. The reverse implication is clear.

ThEOREM 2.7. We have $Z\left(R\left[x_{1}, x_{2}, \cdots\right]\right)=Z(R)\left[x_{1}, x_{2}, \cdots\right]$ for any $\operatorname{ring} R$ 。

Proof. Let $S=R\left[x_{1}, x_{2}, \cdots\right]$. Let $a_{1}, \cdots, a_{n}$ denote the coefficients of some polynomial $p$ in $S$. If each $\alpha_{i} \in Z(R)$ for $1 \leqq i \leqq n$ then the 
finite intersection $\bigcap r\left(a_{i}\right)$ for $1 \leqq i \leqq n$ is essential in $R$. Clearly $r(p)$ contains $\bigcap r\left(a_{i}\right)\left[x_{1}, x_{2}, \cdots\right]$ for $1 \leqq i \leqq n$ and $\bigcap r\left(a_{i}\right)\left[x_{1}, x_{2}, \cdots\right]$ is essential in $S$. Therefore, $Z(R)\left[x_{1}, x_{2}, \cdots\right] \subseteq Z(S)$. Let $h$ be a polynomial in $Z(S)$. Write $h$ as a sum of two polynomials $h_{1}$ and $h_{2}$ such that $h_{1} \in Z(R)\left[x_{1}, x_{2}, \cdots\right]$ and each nonzero coefficient of $h_{2}$ does not belong to $Z(R)$. Also $h_{1}$ and $h_{2}$ are in $Z(S)$. If $h_{2} \neq 0$ then let $a_{n}$ be the leading coefficient of $h_{2}$. Now $a_{n} \in R-Z(R)$ implies $r\left(a_{n}\right) \cap b R^{1}=(0)$ for some nonzero $b$ in $R$ and $r\left(h_{2}\right) \cap b S^{1}=(0)$ a contradiction. Therefore, $h_{2}=0$ and $h \leqq Z(R)\left[x_{1}, x_{2}, \cdots\right]$ which completes the proof.

3. Applications. In this section we extend to polynomial rings certain order theorems of A. W. Goldie and L. Small. Henceforth, assume that $R$ is a ring with unity. A right ideal $K$ is called a right annihilator if $K=r(S)$ for some appropriate subset $S$ of $R$. A ring is called a Goldie ring if it is a right finite dimensional ring which satisfies the maximum condition on right annihilators.

Proposition 3.1. A ring $R$ is a semiprime Goldie ring if and only if $R\left[x_{1}, x_{2}, \cdots\right]$ is.

Proof. It is well known that a semiprime Goldie ring is a semiprime right finite dimensional ring with a zero singular ideal. Let $S=R\left[x_{1}, x_{2}, \cdots\right]$. Theorem 2.6 implies $R$ is right finite dimensional if and only if $S$ is. Theorem 2.5 implies $Z(R)=(0)$ if and only if $Z(S)=(0)$. It is clear that $R$ is semiprime if and only if $S$ is. This completes the proof.

Corollary 3.2. (Small [5]) A ring $R$ is a semiprime Goldie ring if and only if $R\left[x_{1}, x_{2}, \cdots, x_{n}\right]$ is for all $n$.

Proof. The proof is clear.

Theorem 3.3. (R. C. Shock [3]). Let $R$ denote a right finite dimensional ring. A nil subring $S$ is nilpotent in $R$ if and only if the subring $S \cap Z(R)$ is nilpotent. Furthermore, if $Z(R)$ is nilpotent with index $k$ then each nil subring is nilpotent and has index of nilpotency $\leqq k(\operatorname{dim} R+1)$.

\section{Proof. See [3].}

THEOREM 3.4. Let $R$ be a right finite dimensional ring. Let $S=R\left[x_{1}, x_{2}, \cdots\right] . \quad A$ nil subring $K$ is nilpotent in $S$ if and only if $K \cap Z(R)\left[x_{1}, x_{2}, \cdots\right]$ is nilpotent. Furthermore, if $Z(R)$ is nilpotent with index then each nil subring is nilpotent in $S$ and has index 
of nilpotency $\leqq k(\operatorname{dim} R+1)$.

Proof. The proof follows immediately from Theorem 3.3, Theorem 2.6, and Theorem 2.7.

For a subset $K$ of a ring $R$ we equate $l(K)$ with the set $\{x \in$ $R: x k=0$ for all $k \in K\}$.

We refer to [7] for notation and definitions used below.

THEOREM 3.5. (L. Small). Let $N$ denote the prime radical of $R$. Assume $R$ satisfies the following properties:

(1) The prime radical $N$ of $R$ is nilpotent and $R / N$ is a Goldie ring.

(2) Each factor ring $R / T_{k}$ where $T_{k}=l\left(N^{k}\right) \cap N$ is right finite dimensional for all nonnegative integers $k$.

(3) There is an exhaustive set $S(M)$ consisting of nonzero divisors of $R$. Then $R$ has a classical ring of right quotients which is an Artinian ring.

Proof. See [5], [6], and [7].

THEOREM 3.6. If $R$ has a classical ring of right quotients which is Artinian then so does $R\left[x_{1}, x_{2}, \cdots\right]$.

Proof. Let $S=R\left[x_{1}, x_{2}, \cdots\right]$ and we verify that $S$ satisfies the hypothesis of Theorem 3.5. Let $N(R)$ be the prime radical of $R, N(S)$ the prime radical of $S$. It is wellknown that $N(S)^{k}=N(R)^{k}\left[x_{1}, x_{2}, \cdots\right]$ for all $k \geqq 1$. Since $R / N(R)$ is a semiprime Goldie $\operatorname{ring}, S / N(S)$ which is isomorphic to $R / N(R)\left[x_{1}, x_{2}, \cdots\right]$ is also a semiprime Goldie ring by Proposition 3.1. Let $T_{k}=l\left(N(S)^{k}\right) \cap N(S)$ where $k$ is a nonnegative integer. If $p \in T_{k}$ then $p y=0$ for all $y \in N(R)^{k}$. The coefficients of $p$ lie in $l\left(N(R)^{k}\right) \cap N(R)$ and hence $T_{k}=\left(l\left(N(R)^{k}\right) \cap N(R)\right)\left[x_{1}, x_{2}, \cdots\right]$. The factor ring $S / T_{k}$ is right finite dimensional because $S / T_{k}$ is isomorphic to $R / l\left(N(R)^{k}\right) \cap N(R)\left[x_{1}, x_{2}, \cdots\right]$ and $R / l\left(N(R)^{k} \cap N(R)\right.$ is right finite dimensional. Let $S(M)$ denote the set of polynomials in $S$ whose leading coefficients are nonzero divisors. The set $S(M)$ is an exhaustive set in $S$, as the proof is an easy modification of the proof of Lemma 2 and Theorem 2 of [7]. This completes the proof.

Corollary 3.7. (L. Small [5]) If $R$ has a classical ring of right quotients which is Artinian then so does $R\left[x_{1}, x_{2}, \cdots, x_{n}\right]$ for all $n$.

Proof. This is clear. 


\section{REFERENCES}

1. A. W. Goldie, Semiprime rings with the maximum condition, Proc. London Math. Soc., 10 (1960), 201-220.

2. R. E. Johnson, Exlended centralizer of a ring over a module, Proc. Amer. Soc., 2 (1951), 891-895.

3. R. C. Shock, Nil subrings of finite dimensional rings, Submitted.

4. - Ring with finiteness conditions, Doctoral Dissertation at The University of North Carolina at Chapel Hill, 1969.

5. L. W. Small, Orders in Artinian rings, J. Algebra, 4 (1966), 13-14.

6. Correction and Addendum: Orders in Artinian rings, J. Algebra, 4 (1966), 505-507.

7. - Orders in Artinian rings, II. J. Algebra, 9 (1968), 226-273.

Received February 16, 1971 and in revised form July 1, 1971.

SOUTHERN ILLINOIS UNIVERSITY 



\section{PACIFIC JOURNAL OF MATHEMATICS}

\section{EDITORS}

H. SAMELSON

Stanford University

Stanford, California 94305

C. R. HobBY

University of Washington

Seattle, Washington 98105
J. DUGUNDJI

Department of Mathematics

University of Southern California

Los Angeles, California 90007

RICHARD ARENS

University of California

Los Angeles, California 90024

\section{ASSOCIATE EDITORS}

E. F. BECKENBACH

B. H. NeumanN

F WoLF

K. YoshidA

\section{SUPPORTING INSTITUTIONS}

UNIVERSITY OF BRITISH COLUMBIA

UNIVERSITY OF SOUTHERN CALIFORNIA

CALIFORNIA INSTITUTE OF TECHNOLOGY

UNIVERSITY OF CALIFORNIA

MONTANA STATE UNIVERSITY

STANFORD UNIVERSITY

UNIVERSITY OF NEVADA

NEW MEXICO STATE UNIVERSITY

OREGON STATE UNIVERSITY

UNIVERSITY OF OREGON

OSAKA UNIVERSITY

UNIVERSITY OF TOKYO

UNIVERSITY OF UTAH

WASHINGTON STATE UNIVERSITY

UNIVERSITY OF WASHINGTON

$\stackrel{*}{*} \stackrel{*}{*} \stackrel{*}{*}{ }^{*}{ }^{*}$ MMEICAN MATHEMATICAL SOCIETY

NAVAL WEAPONS CENTER

Printed in Japan by International Academic Printing Co., Ltd., Tokyo, Japan 


\section{Pacific Journal of Mathematics}

\section{Vol. 42, No. $1 \quad$ January, 1972}

Tage Bai Andersen, On Banach space valued extensions from split faces ........

David Marion Arnold, A duality for quotient divisible abelian groups of finite

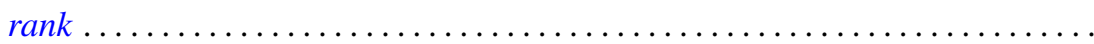

Donald Pollard Ballou, Shock sets for first order nonlinear hyperbolic

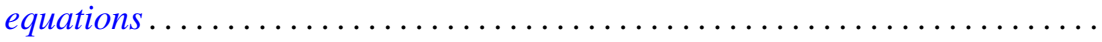

Leon Brown and Lowell J. Hansen, On the range sets of $H^{p}$ functions .........

Alexander Munro Davie and Arne Stray, Interpolation sets for analytic

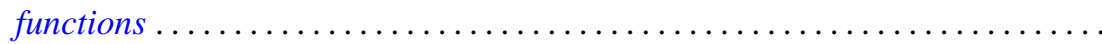

M. G. Deshpande, Structure of right subdirectly irreducible rings. II . . . . . . . . .

Barry J. Gardner, Some closure properties for torsion classes of abelian

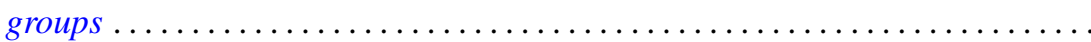

Paul Daniel Hill, Primary groups whose subgroups of smaller cardinality are

direct sums of cyclic groups . . . . . . . . . . . . . . . . . . .

Richard Allan Holzsager, When certain natural maps are equivalences .........

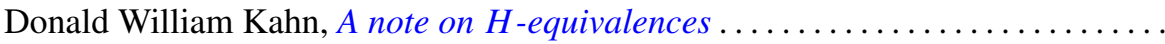

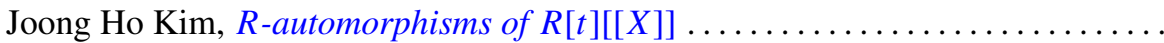

Shin'ichi Kinoshita, On elementary ideals of polyhedra in the 3-sphere.........

Andrew T. Kitchen, Watts cohomology and separability...

Vadim Komkov, A technique for the detection of oscillation of second order

ordinary differential equations .

Charles Philip Lanski and Susan Montgomery, Lie structure of prime rings of characteristic 2

Andrew Lenard, Some remarks on large Toeplitz determinants . .

Kathleen B. Levitz, A characterization of general Z.P.I.-rings. II .

Donald A. Lutz, On the reduction of rank of linear differential systems

David G. Mead, Determinantal ideals, identities, and the Wronskian ...

Arunava Mukherjea, A remark on Tonelli's theorem on integration in product

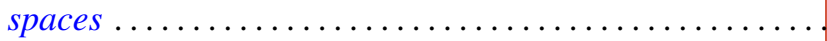

Hyo Chul Myung, A generalization of the prime radical in nonassociative rings.

John Piepenbrink, Rellich densities and an application to unconditionally nonoscillatory elliptic equations.

Michael J. Powers, Lefschetz fixed point theorems for a new class of multi-valued maps .

Aribindi Satyanarayan Rao, On the absolute matrix summability of a Fourier

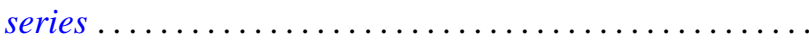

T. S. Ravisankar, On Malcev algebras ......................... 227

William Henry Ruckle, Topologies on sequences spaces . . . . . . . . . . . . . 235

Robert C. Shock, Polynomial rings over finite dimensional rings . . . . . . . . . 251

Richard Tangeman, Strong heredity in radical classes . . . . . . . . . . . . . . 259

B. R. Wenner, Finite-dimensional properties of infinite-dimensional spaces . . . . 267 